EUROPEAN ORGANIZATION FOR NUCLEAR RESEARCH

European Laboratory for Particle Physics

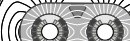

(1) (3)

Large Hadron Collider Project

LHC Project Report 124

\title{
Dynamic Effects and their Control at the LHC
}

\author{
R. Bailey+, F. Bordry+, L. Bottura**, P. Burla*, P. Collier+, K.Henrichsen**, \\ J.P. Koutchouk+, R. Lauckner+, R. Parker+, J. Pett+, P. Proudlock*, \\ H. Schmicklert, R. Schmidt**, L. Walckiers**, R. Wolf**
}

\begin{abstract}
Tune, chromaticity and orbit of the LHC beams have to be precisely controlled by synchronising the magnetic field of quadrupole, sextupole and corrector magnets. This is a challenging task for an accelerator using superconducting magnets, whose field and field errors will have large dynamic effects. The accelerator physics requirements are tight due to the limited dynamic aperture and the large energy stored in the beams. The power converters need to be programmed in order to generate the magnetic functions with defined tolerances.

During the injection process and the energy ramp the magnetic performance cannot be predicted with sufficient accuracy, and therefore real-time feedback systems based on magnetic measurements and beam observations are proposed. Beam measurements are used to determine a correction factor for some of the power converters. From magnetic measurements the excitation of small magnets to compensate the sextupolar $\left(b_{3}\right)$ and decapolar $\left(b_{5}\right)$ field components in the dipole magnets will be derived. To meet these requirements a deterministic control system is envisaged.
\end{abstract}

${ }^{*} \mathrm{AC},{ }^{* *}$ LHC Division, +SL Division

Presented at 1997 Particle Accelerator Conference, Vancouver, 12-16 May 1997.

Administrative Secretariat

LHC Division

CERN

CH - 1211 Geneva 23

Switzerland

Geneva, 9 July 1997 



\title{
Dynamic Effects and their Control at the LHC
}

\author{
R.Bailey, F.Bordry, L.Bottura, P.Burla, P.Collier, K.Henrichsen, J.P. Koutchouk, R.Lauckner, \\ R.Parker, J.Pett, P.Proudlock, H.Schmickler, R.Schmidt, L.Walckiers, R.Wolf \\ (CERN, Geneva, Switzerland)
}

\section{ABSTRACT}

Tune, chromaticity and orbit of the LHC beams have to be precisely controlled by synchronising the magnetic field of quadrupole, sextupole and corrector magnets. This is a challenging task for an accelerator using superconducting magnets, whose field and field errors will have large dynamic effects. The accelerator physics requirements are tight due to the limited dynamic aperture and the large energy stored in the beams. The power converters need to be programmed in order to generate the magnetic functions with defined tolerances. During the injection process and the energy ramp the magnetic performance cannot be predicted with sufficient accuracy, and therefore realtime feedback systems based on magnetic measurements and beam observations are proposed. Beam measurements are used to determine a correction factor for some of the power converters. From magnetic measurements the excitation of small magnets to compensate the sextupolar $\left(b_{3}\right)$ and decapolar $\left(b_{5}\right)$ field components in the dipole magnets will be derived. To meet these requirements a deterministic control system is envisaged.

\section{INTRODUCTION}

This paper summarises some of the findings of the LHC Dynamic Effects Working Group. It also reflects the discussions and conclusions of a three day workshop held at CERN in February 1997 [1] [2].

The injection and acceleration of the LHC proton beams without particle losses and emittance growth requires an accurate control of the beam parameters. The value of the betatron tune is about 63 units and needs to be controlled to a level of 0.003 (for ultimate performance) [3]. Orbit excursions should be limited to less than $0.5 \mathrm{~mm}$ (rms value of the closed orbit). The chromaticity should be limited to some units (the chromaticity is defined as the change of tune with energy). Therefore the magnetic fields of the dipole, quadrupole, sextupole and corrector magnets need to be precisely synchronised during the energy ramp. This must be done in the presence of varying response times and transfer functions of the power converters and magnets. For an accelerator with superconducting magnets, such as the LHC, the synchronisation is a particular challenge due to large variations in the field and field errors caused by coupling currents and persistent currents within the superconducting cable. Dynamic effects include changes of the magnetic field and its multipoles even at constant excitation current, and changes of the field depending on the speed of the ramp as well as on the powering history.

\section{DYNAMIC EFFECTS IN SUPERCONDUCTING MAGNETS}

Persistent current effects in superconducting magnets produce large allowed multipole components in the magnetic field. The phenomenon is strongest at low field and is highly non-linear in magnet excitation and temperature.

It was first observed at the TEVATRON that multipole fields exhibit slow time drifts even if the current is kept constant [4]. When the energy ramp is resumed, a rapid change of the multipole fields is observed ("snap-back"). Although it has been over 10 years since this phenomenon was first observed, the understanding of the time dependence of persistent currents is not complete. For the LHC prototype dipole magnets the results from detailed measurements of persistent current decay and snap-back are documented in [5] [6]. The decay at stable current and snap-back (Fig.1) of the fundamental and multipole components depend on the previous powering history of the magnet and are not, at present, easy to predict. Theories have been put forward for this phenomena (interaction of the internal field with the cable magnetisation) suggesting that there is hope for a better predictability of these errors in the future [5] [7]. From a practical point of view consistent behaviour can be best achieved by a precise and well defined cycle of the main magnets, as in the operational procedures for the TEVATRON, where the magnets are cycled six times before injection. Because of the time involved (about one hour for a complete cycle) multiple-cycling before injection can not be envisaged for the LHC. In the TEVATRON and at HERA the beam is only sensitive to the chromaticity due to sextupolar field components created by the persistent currents in the dipole magnets. For the LHC, decapolar as well as sextupolar field components in the dipole magnets require correction.

Dynamic effects during the ramp, due to coupling currents between the strands inside the cable, are now well understood [8]. By controlling the inter-strand contact resistance in the superconducting cable and limiting its spread it is expected to achieve a good reproducibility of the coupling currents for the series of LHC dipole magnets.

\section{MAGNETS AND POWERING OF LHC}

The large size and certain constraints imposed by the use of the existing infrastructure (for example the LEP tunnel and the existing underground structures) have resulted in an electrical segmentation of the machine into eight sectors requiring tracking amongst sectors as well as families of magnets. Each of the eight LHC sectors has one family of main dipole magnets and separately powered main 


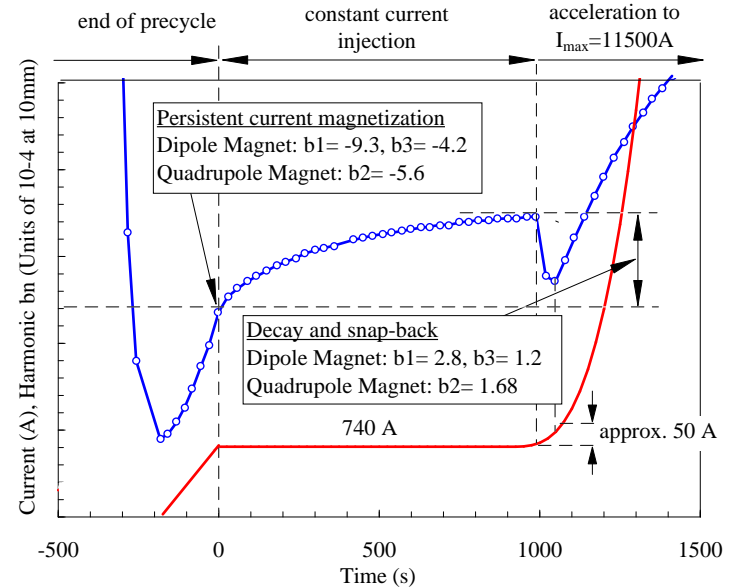

Figure 1: Schematic view of current and magnetic field versus time. The field decays during the injection plateau, and is re-established suddenly at the start of the ramp

quadrupoles (QD and QF). A dipole orbit corrector is associated with each quadrupole aperture. For the correction of the betatron tunes trim quadrupole magnets are foreseen, since a fast correction of the tune with the lattice quadrupoles is difficult to perform, due to the large inductance and therefore long time constant of the magnet chain. Families of tuning quadrupoles and dispersion suppressors are installed at both ends of each sector. Families of chromaticity sextupoles, octupoles and skew-quadrupoles are installed close to the quadrupole magnets in the arcs. Small sextupole and decapole corrector magnets will be installed at the end of each dipole magnet for local compensation of the multipoles in order to maximise the dynamic aperture.

In order to achieve the slow ramp rates as well as for fine adjustments, the main power converters will need a monotonic resolution of $1 \mathrm{ppm}$ [9]. Because of the large hysteresis in the dc magnetisation curves it is important that the overshoot (and undershoot) is zero or at least kept below 5 $\mathrm{ppm}$. Although absolute accuracy is not an issue, a shortterm stability and dynamics of \pm 3 ppm as well as a reproducibility and tracking between sectors of $\pm 5 \mathrm{ppm}$ is required. The requirements, expressed in terms of maximum current, come from the needs at injection. These are 15 times less severe when expressed with respect to the actual current at injection. To meet these requirements, and because of the large electrical time-constants of the main circuits (23000s for the dipole), digital predictive regulation techniques will be used with a 20-bit Sigma-Delta ADC.

\section{ORBIT AND BETATRON TUNE}

The central momentum of the particles is determined by the integral dipole field. The frequency of the RF-system is nearly constant and determines the mean radial orbit. Differences of the integral field of the dipole magnets in the octants need to be smaller than $10^{-4}$ which requires an excellent tracking of the power converters. If this value is exceeded, the orbit is adjusted with correction dipole magnets. During the time to inject beam into the LHC (this will take at least eight minutes) the main dipole field increases slightly due to the decay of the persistent currents. To maintain the energy matching between LHC and SPS (as injector) the average strength of the orbit corrector magnets will be reduced to keep the integral dipole field and therefore the energy constant. At some critical locations, for example close to the collimators, a local orbit feedback is proposed.

To control the betatron tunes to a level of 0.003 the reproducibility of the quadrupole gradient with respect to the particle momentum needs to be better than $40 \mathrm{ppm}$. Since the power converter is always rated with respect to maximum current, a reproducibility of better than $2 \mathrm{ppm}$ would be required. Not only the lattice quadrupoles need to be precisely synchronised to the particle momentum, but also the quadrupoles in the insertions. Due to magnet dynamic effects and power converter tolerances the energy cannot be ramped without correcting the tunes. Therefore on-line tune measurements and corrections are required at an early stage of the LHC operation using the tuning quadrupoles. The main dipole and quadrupole magnets will follow a predefined current ramp.

\section{CHROMATICITY AND HIGHER ORDER MULTIPOLES}

The chromaticity is generated by the optics and by the sextupolar field component $\left(b_{3}\right)$ in the dipole magnets. The sextupole component of the persistent currents in the dipole magnets generates a chromaticity of about 550 units. The natural chromaticity is about 100 units. During the time to inject beam into the LHC the chromaticity changes slowly due to the decay of the persistent currents. A correction is performed with the sextupole correctors at the end of the dipoles, by either using a measurement of $b_{3}$ or a model for the persistent current decay. Since $b_{3}$ needs to be corrected to the $1 \%$ level or better, the required accuracy might not be achieved. Chromaticity measurements on the injection plateau will complement the magnetic measurements.

At the start of the energy ramp the snap-back leads to a chromaticity change of about 150 units, the time for this change depends on the initial speed of the ramp. It has been estimated, that after a field increase of about $35 \mathrm{mT}$ the snap back is finished. This corresponds to a current increase of about $50 \mathrm{~A}$. It is proposed to start the ramp very slowly so as to minimise the rate of change in the field and field errors. The sextupole corrector magnets will be ramped according to the results of magnetic measurements in the reference magnets. Again, chromaticity measurements can be performed for fine adjustments. Another option is to increase the current in small steps of, say, $0.5 \mathrm{~A}$, and to measure and adjust the chromaticity for each step.

The control of the decapole corrector magnets relies on measurements of the $b_{5}$ component in reference magnets. At injection, the current will be adjusted to compensate the 
$b_{5}$ component in the dipole magnets. During the snap-back, the corrector magnets have to be ramped, again based on magnetic measurements.

Schemes of injection on a very slow ramp to avoid the snap-back have also been considered but have no priority for the moment.

\section{REFERENCE MAGNETS}

The present layout of the LHC foresees a set of reference magnets connected in series with each of the main power converters of the eight separately powered sectors of the machine ( 8 dipole and 8 quadrupole magnets). The reference magnets were to provide the main observables within the machine for its control. However, it became apparent that they would not be able to achieve completely this role. Further reliance needed to be placed on good power converter performance where effects are reproducible and beam observation where this is not the case. Considering:

- the expected performance of the power converters;

- the availability of comprehensive beam observation (eventually on-line);

- the operating principle of correcting all dynamic effects with correcting magnets;

it is proposed to install the reference magnets remotely in a surface building in which the magnetic measurements will be made. There should be one reference magnet per type of dipole and one per type of quadrupole (total of about 6). To allow additional verification within the machine, these remote reference magnets should be complemented by flux-loops measuring the fundamental fields of the dipoles and quadrupoles of the machine magnets of each sector [10]. This results in a much simplified reference magnet system. It is accessible, can be developed from the magnet test benches, is flexible and can be used on-line to assist with machine operation or off-line for new developments. The disadvantages are associated with recovery from a failure of the powering or cryogenics systems and the fact that the remote infrastructure becomes important to machine operation until such time as it is not needed on-line.

\section{BEAM INSTRUMENTATION AND CONTROLS}

For the control of the LHC in presence of dynamic effects the measurement of orbit, tune, coupling and chromaticity is of prime importance. Fast orbit acquisition and good orbit correction software will be needed already at the commissioning stage. New techniques to observe coupling, tune and chromaticity need to be investigated with a view to use these signals for on-line corrections should they prove suitable. Although it is not clear today which parameters require on-line control, it is recommended that the machine is prepared to accept slow feedback as far as the control system, power converters and RF systems are concerned.
Best estimates suggest a rate up to a few $\mathrm{Hz}$, but this will depend, for instance, on the exact magnet ramping speed. The speed of the ramp will depend on the time required to measure and correct tunes, orbit and chromaticity. Therefore field and beam instrumentation should be suitable for inclusion in such a feedback system. Pilot projects are proposed for the SPS, which will concern mainly studies on tune and chromaticity control.

\section{CONCLUSION}

The Working Group on Dynamic Effects has been a very useful forum for discussions among specialist in different domains (magnet technology, beam dynamics, beam instrumentation, power converter, controls and operation). During these discussions, the accelerator physics requirements and specifications for various hardware have been established along with possible scenarios for operation. Considering the present understanding of the behaviour of superconducting magnets it is believed that the dynamic effects of LHC can be controlled using main power converters of reasonable precision ( $1 \mathrm{ppm}$ resolution, $\pm 3 \mathrm{ppm}$ short-term stability), benefiting from on-line magnetic measurements from a limited number of reference magnets. This will require a deterministic control system. For ultimate performance, however, the development of beam instrumentation suitable for feedback loops needs to be undertaken.

\section{ACKNOWLEDGEMENT}

We would like to thank all participants to the 'LHC Workshop on Dynamic Effects and Their Control', in particular the representatives from DESY (HERA), FERMILAB (TEVATRON) and BNL (RHIC).

\section{REFERENCES}

[1] R. Schmidt, Progress Report from the Dynamic Effects Working Group, LHC Project Note 67, October 1996

[2] P. Proudlock, Summary of the LHC Workshop on Dynamic Effects, LHC Project Report 99, March 1997

[3] J.P. Koutchouk et al., A Robust and Flexible Lattice for LHC, these proceedings

[4] D. Finley, PAC 87, Washington DC, USA, 16-19 March 1987

[5] L. Bottura et al., Field Errors Decay and "Snap-Back" in the LHC Model Dipoles, ASC'96, Pittsburgh, PA, USA, 25-30 Aug 1996

[6] L. Walckiers et al., Towards Series Measurements of the LHC Superconducting Magnets, these proceedings

[7] H.Mess et al., Superconducting Accelerator Magnets, World Scientific, 1996

[8] A.P. Verweij, R. Wolf, Field Errors due to Inter-Strand Coupling Currents.., Internal Note CERN-AT-MA/AV 94-97, March 1994

[9] I. Barnett et al, A strategy for controlling the LHC magnet currents, EPAC, Barcelona, Spain, June 10-14, 1996

[10] K. Henrichsen et al., Proposal for in-situ measurements of dynamic effects in the LHC lattice magnets, LHC Project Report 89, January 1997 